\title{
Agents Displacement in Arbitrary Geometrical Spaces An Evolutionary Computation based Approach
}

\author{
Francesco D’Aleo ${ }^{1}$, Fabio D'Asaro ${ }^{1}$, Valerio Perticone ${ }^{1}$, Giovanni Rizzo ${ }^{1}$ and Marco Elio Tabacchi ${ }^{1,2}$ \\ ${ }^{I}$ Dip. di Matematica ed Informatica, Università degli Studi di Palermo, Palermo, Italy \\ ${ }^{2}$ Ed Istituto Nazionale di Ricerche Demopolis, Demopolis, Italy
}

Keywords: Agent-based Modeling, Optimization.

\begin{abstract}
In many different social contexts, communication allows a collective intelligence to emerge. However, a correct way of exchanging information usually requires determined topological configurations of the agents involved in the process. Such a configuration should take into account several parameters, e.g. agents positioning, their proximity and time efficiency of communication. Our aim is to present an algorithm, based on evolutionary programming, which optimizes agents placement on arbitrarily shaped areas. In order to show its ability to deal with arbitrary bi-dimensional topologies, this algorithm has been tested on a set of differently shaped areas that present concavities, convexities and obstacles. This approach can be extended to deal with concrete cases, such as object localization in a delimited area.
\end{abstract}

\section{INTRODUCTION}

In an agent simulation, just as in real social contexts, communication among individuals is one of those characteristics that can enhance survival as well as performances fitness-wise (Caci et al., 2011, Cardaci et al., 2013, Tabacchi et al., 2010, Villata et al., 2012). Communication allows a collective intelligence to emerge, as it has been shown by research on insects and using animat (Bonabeau et al., 1999).

In many scenarios it is necessary to transmit messages to a number of distinct intermediaries (while other information can be delivered to many agents at the same time using broadcast mode, Terna \& Taormina, 2007), due to the distance between individuals. The concept of distance itself can be put into question: in nature, distances are usually regarded as "physical" distances, while in other scenarios this concept can be readily extended; for instance, consider the cost of a link between two computers (Barabási, 2002), the energy cost of a biological link between cells (Albert, 2005), or even between concepts (Petrou et al., 2010). Further problems can be caused by the presence of natural or artificial obstacles between two individuals, or even by a limited channel capacity.

In our proposed scenario, several agents inhabit an arbitrarily shaped environment populated by obstacles, and they can only communicate through intermediaries lying at some maximal distance one from another; each of such intermediaries is able to receive messages from several sources and forward them to other individuals in the designed area. This is a broadcast kind of transmission: in order to reach the destination, a single individual forwards the received message to several intermediaries, ensuring some desirable characteristics such as the robustness and reliability of the ensuing network.

The number of agents in such a network is usually variable, and it is necessary to consider the possibility that some specific agents could reach a huge number of other agents lying at a reasonable distance, and at the same time to have a low number of errors due to the transmission medium and to episodes such as the temporary inactivation of an agent or even its permanent removal from the network. Therefore it is not out of place to draw an analogy with social networks dynamics: in our case, intermediaries behave just like hubs making possible for distant nodes, which would otherwise have difficulties to reach each other, to communicate.

A central parameter to consider, if we are to assess the network evolution in time, is the minimum number of agents needed to reach every agent in the network; finding an optimal value without introducing errors or noise in the signal is a

198 D'Aleo F., D’Asaro F., Perticone V., Rizzo G. and Elio Tabacchi M. 
notoriously hard problem (Tabacchi \& Termini, 2011). On the other hand, if we consider the network arrangement in space, the topology of the environment - that is, the absolute (with respect to space) and relative (with respect to other agents) position of each agent - has to be carefully taken into account. Optimal and sub-optimal solutions to well-known and highly idealized situations are already available in literature, including Steiner's Tree (Hwang et al., 1992); however, it is harder to deal with harsher, possibly non-convex, noncontiguous, and/or arbitrarily complex areas.

In these cases, a soft-computing technique (Seising and Tabacchi, 2013) such as evolutionary programming (Michalewicz, 1996) could provide sub-optimal solutions in a reasonable amount of time. Evolutionary algorithms in general, and especially genetic algorithms, are already successfully applied to a huge number of optimization problems and they are proven of being capable to efficiently find "good" solutions, i.e. solutions that closely approximate the best one.

In this work we propose a preliminary version of an evolutionary algorithm, which serves to optimize the number and position of agents in arbitrarily shaped areas, possibly populated by obstacles. Results have shown how the algorithm satisfies suboptimality criteria as well as strong connectivity requirements and were also obtained in an efficient way. Even more, the algorithm tries to optimize the number of agents, removing the superabundant ones from the map.

This article is organized as follows: in the next section we shall describe in detail the problem we are concerned with and the approach we chose to face it. In the third section we shall present the algorithm as we implemented it, while analyzing the principal mechanisms regulating agents and entities of the simulation. Last section is devoted to conclusions and to possible further developments of this work. Some alternative methodologies and different approaches to this very problem are discussed and it will be pointed out how this work can be applied to problems of a very different nature than the one considered here.

\section{METHODOLOGY}

In the study and analysis of an ideal network, it is crucial to focus on the first stages of its creation, since the initial topology can have a severe impact on network functionalities and evolution, as well as on its principal entities, i.e. those nodes establishing interconnections among peers.

Amongst the fundamental aspects to be considered we should mention the minimization of the number of entities involved in the peercommunication process, which plays an important role during the construction stage. In fact, an excessive number of agents can lead to some inconveniences (already well-known in information theory) such as information redundancy and corruption due to signal degradation. However, decreasing the number of entities should not compromise the connection between them therefore it turns out to be indispensable to evaluate the proportion between number of nodes and available space.

Another point that should be carefully taken into account is that network topology could contemplate the presence of hubs, which play a fundamental role for the reasons discussed in the introduction; however, the number of hubs in a network should not exceed a critical limit, since otherwise we would get an inefficient network due to the redundancy of some connections.

Some mathematical models to deal with canonically shaped areas are already known in literature; they allow to find the "best" (with regard to communication efficiency and total number of entities) way of positioning entities in such highly idealized scenarios, though this formally restricts the possible choices by presenting characteristics that are not modifiable by those entities moving in the arbitrarily shaped spaces.

Considering all the limits and problems arising in such situations, it comes as no surprise that finding the optimal solution is a computationally unfeasible problem. However, we can find suboptimal solutions in a reasonable amount of time using evolutionary programming techniques. In particular, in the next section we shall introduce and discuss a procedure that employs an evolutionary algorithm augmented by hill-climbing, of which many variants are available.

Even though a full detailed description of the algorithm is the focus of the next chapter, we want to highlight that the algorithm plays a crucial role because of its capacity of satisfying all the requisites mentioned before. This very algorithm acts as a leader, imposing a direction for the entities to move, without asking them to act in a precise way. The leader also evaluates the solution at each stage of the process, using a fitness function, which is based on an evaluation of the total area covered by each agent.

Some motivation for the leader to be 
implemented through a genetic algorithm comes from the fact that similar approaches were already successfully used in various areas such as wire routing, scheduling, adaptive control, game playing, cognitive modeling, transportation problems, traveling salesman problems, optimal control problems, database query optimization (for a review see Michalewicz, 1996).

Single agents, on the other hand, are implemented through the already mentioned hillclimbing algorithm, that is, a greedy algorithm which at each iteration chooses the best state, leading every time to a better (or, at least, nonworse) solution.

Unlike a gradient-descending technique, we chose the possibility of introducing a tabu search variant to avoid repeated actions.

\section{ALGORITHM}

The idea behind our algorithm is to initially set up the map in a way derived from the optimal solution for a canonical shape surrounding the area we are considering. For such shapes we can indeed use some well known algorithms about Convex Hull problem in a bi-dimensional plane. Such algorithms are based on several differential and computational geometry theorems (Vazirani, 2001). In particular, Groemer's theorem (Groemer, 1960) establishes a relation between an area and the number of nodes that are needed to cover (compact or convex) parts of it. However, efficient algorithms in the more general case of non-convex, non-compact areas are not known.

In this work, the "optimal solution" is considered as a starting point from which it is then possible to calculate, through refinements, the desired suboptimal solution for the real area in consideration. The refining procedure consists of moving the agents in every possible way in a pre-defined neighborhood, while verifying whether the new placements leads to an improvement of the general connectivity status. More precisely, agents are able to move in their neighborhood according to a turnbased mechanism.

At each turn the leader, which cannot be identified with any agent and which therefore constitutes an external entity, evaluates whether it is necessary to order a movement or not. Such broadcast communication forces all the agents to make a displacement, providing them useful information about their neighborhood.

It should be carefully noticed that in some
Table 1: Highlights of the algorithm.

1. Set up the agents in a way derived from the optimal solution, as suggested by Groemer's Theorem;

2. Moving the agents in every possible way in a pre-defined neighborhood and recalculate the fitness function;

3. Repeat step two until find the sub-optimal solution. Any configurations loop are avoided using the tabu search;

4. Remove one of the agents and recalculate the fitness function;

5. Repeat step four until find a configuration with a fitness function better or worse, however, not more than a certain tolerance $(5 \%)$ compared to the solution of the previous iteration.

situations the presence of an agent could turn out to be redundant; these superabundant agents should be removed from the map, since every such agent bears a cost.

Our leader can take into consideration this important factor by progressively removing redundant components, i.e. those that carry no significantly contribution to the improvement of the global solution. The "greedy" procedure described in steps $4-5$ is clearly order-dependent since the order in which the antennas are sequentially taken off might affect the final result. In fact, we could perform an exhaustive (hence, order-independent) search of the optimal set of antennas to be removed instead. However, such a process would be unfeasible computationally wise, while our "greedy" procedure, even though order-dependent, is more efficient and approximates the theoretical optimum with sufficient precision. The presence of a leader provides the possibility of reducing the network's size without having to care about conflicts between agents and/or spontaneous deletion of single nodes.

The main parameters involved in this computation are: the initial distance between agents, the minimum distance allowed between different agents, the size of the neighborhood. These parameters are set at the beginning of the simulation and remain fixed during the entire execution of the procedure. The leader only considers the first of these three variables, while the latter two are broadcasted to each single agent.

Simulation halts whenever the leader stops communicating relevant information to the agents. Such process is asynchronous due to the indirect nature of the communication. Once this organizational process is over, agents can go on exchanging information in an efficient way, expPloiting the new optimized network topology 
Table 2: Results obtained from pseudo-random generated samples. In the second row the area covered by agents is shown using Groemer's Theorem. Each grey level represents the number of agents covering the underlying area, according to the following scale: $\square=0, \square=1, \square=2, \square=3, \square=4, \square=5$ or more. In the third row the disposition of agents after optimization is shown.

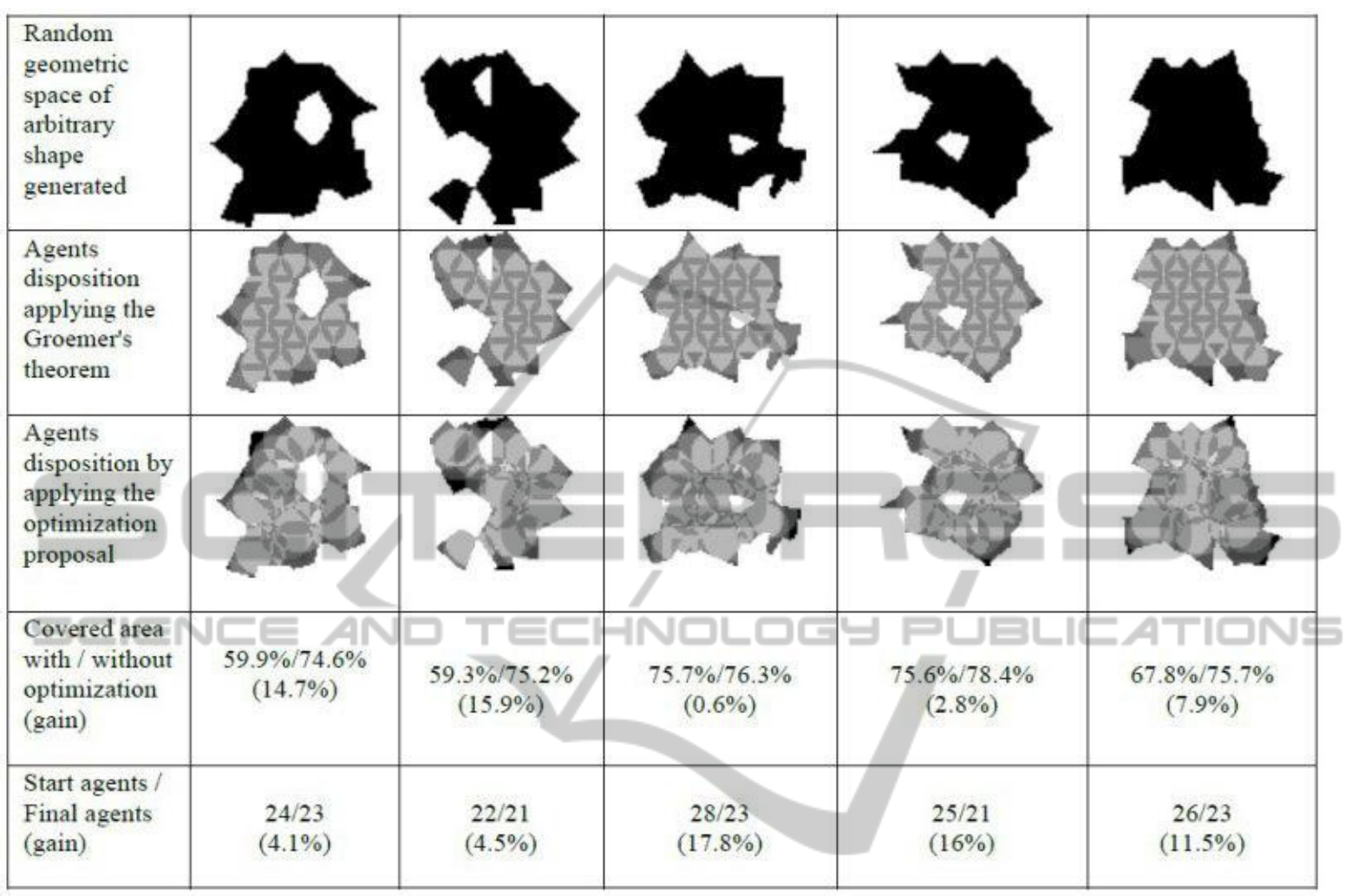

\section{CONCLUSIONS}

A first collection of tests carried out in a simulated environment confirmed the soundness of our algorithm. This reinforces the hypothesis about the validity of abstract mathematical models when they are applied to social areas such as information exchange.

If a suitable set of parameters is chosen, this approach can be readily extended to other fields, e.g. object localization in a three-dimensional environment.

Further developments of this work could contemplate the possibility of putting aside the leader and making single agents independent. In this case they will be free to decide action timing and to start/resolve conflicts to eliminate superfluous agents. Obviously this would lead to a new set of (computational) issues related to the observance of global constraints, which however might be conveniently handled through information exchange between agents.
Furthermore, fuzzy sets theory can be fruitfully applied to this framework by using a suitable membership function wherever required (D'Asaro et al., 2013a, D'Asaro et al., 2013b). In this way, it is possible to find a suitable solution even when an external constraint prevents the fitness function from being maximized.

Table 3: Aggregated results from a pseudo-random generated sample.

\begin{tabular}{|l|c|}
\hline Sample & $\begin{array}{c}\mathrm{N}= \\
1.000\end{array}$ \\
\hline $\begin{array}{l}\text { Average (Standard Deviation) of the } \\
\text { coverage for 3 or more agents using the }\end{array}$ & $\begin{array}{c}62.29 \% \\
(8.53 \%)\end{array}$ \\
\hline $\begin{array}{l}\text { Aroemer's theorem } \\
\text { coverage for 3 or more agents using the } \\
\text { algorithm proposed }\end{array}$ & $\begin{array}{c}71.17 \% \\
(5.94 \%)\end{array}$ \\
\hline $\begin{array}{l}\text { Average (Standard Deviation) of the number } \\
\text { of removed agents }\end{array}$ & $\begin{array}{c}10.82 \% \\
(5.66 \%)\end{array}$ \\
\hline
\end{tabular}




\section{REFERENCES}

Albert, R. (2005). Scale-free networks in cell biology. Journal of cell science, 118(21):4947-4957.

Barabási, A. L. (2002). Linked: The New Science of Networks. Basic Books.

Bonabeau, E., Dorigo, M., Theraulaz, G. (1999). Swarm intelligence. Oxford.

Caci, B., Cardaci, M., Tabacchi, M. E. (2011). Facebook: topology to personality and back - an actor-based simulation. In Kokinov, B., Karmiloff-Smith, A., and Nersessian, N. (eds.) European Perspectives on Cognitive Science. New Bulgarian University Press.

Cardaci, M., Fiordispina, M., Perticone, V., Tabacchi, M.E. (2013). Reti sociali, informazioni individuali. Una simulazione basata su agenti, metodologia fuzzy e Computing With Words. In Auricchio A., Cruciani M., Rega A., Villani M. (eds.) Atti del Convegno AISC 2013, pp. 71-77.

D'Asaro, F. A., Perticone, V., \& Tabacchi, M. E. (2013a, August). A fuzzy methodology to alleviate information overload in eLearning. In 8 th conference of the European Society for Fuzzy Logic and Technology (EUSFLAT-13). Atlantis Press.

D'Asaro, F. A., Perticone, V., Tabacchi, M. E., \& Termini, S. (2013b). Reflections on Technology and Human Sciences: rediscovering a common thread through the analysis of a few epistemological features of fuzziness. Archives for the Philosophy and History of Soft Computing, (1).

Groemer, H. (1960). Über die einlagerung von kreisen in einen konvexen bereich. Mathematische Zeitschrift, 73(3):285-294.

Hwang, F., Richards, D., and Winter, P. (1992). The Steiner Tree Problem. Annals of Discrete Mathematics. Elsevier Science.

Michalewicz, Z. (1996). Genetic algorithms + data structures $=$ evolution programs. Springer.

Petrou, M., Tabacchi, M. E., Piroddi, R. (2010). Networks of Concepts and Ideas. The Computer Journal, 53(10):1738--1751.

Russell, S., Norvig, P. (2010). Artificial Intelligence: A Modern Approach. Prentice-Hall.

Seising, R., Tabacchi, M. E. (2013). A very brief history of soft computing. In Pedrycz, W. and Reformat, M. (eds.) 2013 Joint IFSA World Congress NAFIPS Annual Meeting. IEEE SMC.

Tabacchi, M. E., Caci, B., Cardaci, M. (2010). Comportamenti individuali e connettivi in Facebook: uno studio simulativo. In Ferrari, G., Bouquet, P., Cruciani, M., and Giardini, F. (eds.) Pratiche della Cognizione - Atti del Settimo Convegno Nazionale di Scienze Cognitive, pp. 266--270. AISC.

Tabacchi, M. E., Termini, S. (2011). Measures of fuzziness and information: some challenges from reflections on aesthetic experience. In Proceedings of WConSC 2011.

Terna, P., Taormina, R. (2007). Modelli di simulazione con agenti intelligenti: il sorprendente mondo dei camaleonti. In Sistemi Intelligenti, vol. 19, pp. 391-426. Il Mulino.

Vazirani, V. V. (2001). Approximation algorithms. Springer.

Villata, S., Falcone, R., Da Costa Pereira, C., Castelfranchi, C., Tettamanzi, A., Paglieri F. (2012). Comunicazione e fiducia: un modello ad agenti su qualità delle informazioni e valutazione delle fonti. In Paglieri, F. (ed.) Sistemi Intelligenti, vol. 24, pp. 559-579. Il Mulino.

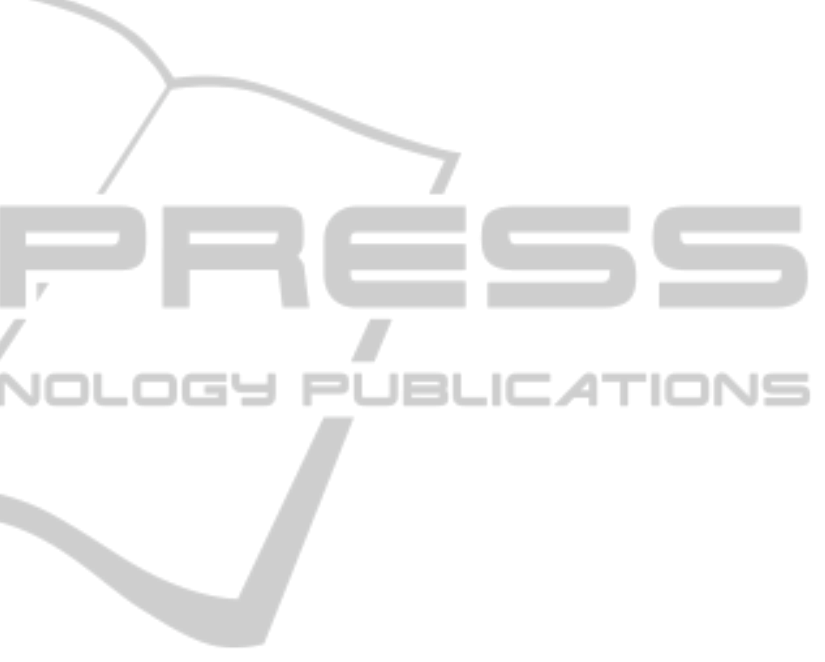

